2015-04-03

$<i>$ The Space Between Us $<$ i $>$ :

Evaluating a multi-user affective

brain-computer music interface

\title{
Eaton, J
}

http://hdl.handle.net/10026.1/6520

\subsection{0/2326263x.2015.1101922}

Brain-Computer Interfaces

Informa UK Limited

All content in PEARL is protected by copyright law. Author manuscripts are made available in accordance with publisher policies. Please cite only the published version using the details provided on the item record or document. In the absence of an open licence (e.g. Creative Commons), permissions for further reuse of content should be sought from the publisher or author. 


\title{
The Space Between Us: Evaluating a multi-user affective brain-computer music interface
}

\author{
Joel Eaton, Duncan Williams \& Eduardo Miranda
}

To cite this article: Joel Eaton, Duncan Williams \& Eduardo Miranda (2015) The Space Between Us: Evaluating a multi-user affective brain-computer music interface, Brain-Computer Interfaces, 2:2-3, 103-116, DOI: 10.1080/2326263X.2015.1101922

To link to this article: http://dx.doi.org/10.1080/2326263X.2015.1101922

\section{Published online: 13 Nov 2015.}

\section{Submit your article to this journal $\square$}

Џ Article views: 1

Q View related articles ¿

View Crossmark data ¿ 


\title{
The Space Between Us: Evaluating a multi-user affective brain-computer music interface
}

\author{
Joel Eaton*, Duncan Williams and Eduardo Miranda \\ Interdisciplinary Centre for Computer Music Research, Plymouth University, Plymouth, UK
}

(Received 8 April 2015; accepted 28 September 2015)

\begin{abstract}
Music as a mechanism for neuro-feedback presents an interesting medium for artistic exploration, especially with regard to passive BCI control. Passive control in a brain-computer music interface (BCMI) provides a means for approximating mental states that can be mapped to select musical phrases, creating a system for real-time musical neuro-feedback. This article presents a BCMI for measuring the affective states of two users, a performer and an audience member, during a live musical performance of the piece titled The Space Between Us. The system adapts to the affective states of the users and selects sequences of a pre-composed musical score. By affect-matching music to mood and subsequently plotting affective musical trajectories across a two-dimensional model of affect, the system attempts to measure the affective interactions of the users, derived from arousal and valence recorded in EEG. An Affective Jukebox, the work of a previous study, validates the method used to read emotions across two dimensions in EEG in response to music. Results from a live performance of The Space Between $U_{S}$ indicate that measures of arousal may be manipulated by emotionally charged music, and that measures of valence are less responsive to musical stimuli, across both users. As such, an affective BCMI presents a novel platform for designing individualized musical performance and composition tools where the selection of music can reflect and induce affect in users. Furthermore, an affective channel of communication shows potential for enhancing collaboration in music-making in a wider context, for example in the roles of therapist and patient.
\end{abstract}

Keywords: brain-computer interface (BCI); music feedback; affective states; electroencephalogram (EEG); musical performance; passive BCI control

\section{Introduction}

Brain-computer interfacing $(\mathrm{BCI})$ offers an alternative to physical or gestural-based control systems as it provides a user with a platform for controlling computer-based systems with the sole input of their brainwaves. This has implications for users with serious motor function impairment, or other difficulties communicating, by providing a platform for communication or interaction which does not require the user to have physical control.[1] As access to neuro-technology has widened, partly through falling costs of electroencephalogram (EEG) devices targeted at the consumer market, so have the creative approaches in which the tools of $\mathrm{BCI}$ are being applied. Research from an artistic perspective is beginning to look beyond the realms of BCI as assistive technology and instead seeks to build systems that can provide enhanced experiences and benefits to users regardless of physical abilities, particularly with regard to music, in what is known as brain-computer music interfacing (BCMI).[2,3]

Applying brainwave analysis to the control and generation of music is not new. It is over 50 years since Alvin Lucier's seminal performance of Music for Solo Performer.[4] However, in more recent times the tech- niques used to elicit brainwave control and the strategies for mapping control to musical systems have progressed, both in line with advances in the fields of $\mathrm{BCI}$ and computer music. Interested readers should refer to [5] for a retrospective account of brainwaves being harnessed for music-making up until the present day.

BCI control can be separated into two discrete categories of user intention. Active control methods provide explicit control over user selection across a limited range of choices, whereas passive control pertains to implicit measurement, where brainwaves are associated with approximations of a user's mental state without any conscious control on the part of the user.[6,7] This article outlines significant developments in on-going research into the detection and use of affective states (emotional approximations) as a control signal in BCMI systems. Proposals and initial results have been previously demonstrated at computer music conferences in 2014 $[8,9]$ and this article is written after a full implementation of a real-time affective performance has been realized through The Space Between us. Whereas a lot of current research in BCI is currently focused towards improving EEG signals and the classification of data from EEG, this article presents work that focuses on the

*Corresponding author. Email: joel.eaton@postgrad.plymouth.ac.uk 
application of existing BCI methods to new ways of controlling music and exploring musical interactions between more than one participant.

Passive control over music presents an interesting approach to sonifying brain activity especially as a novel means of understanding complex and difficult to interpret data from EEG.[10] Previous studies have indicated that passive music control applications can even help towards improving medical symptoms.[11] A major benefit of passive control in BCMIs is that a user is free from the distractions of active control that might otherwise occupy the user's thoughts and detract from the listening experience, particularly active control that requires attention to a non-musical external stimulus.[2,3] One of the drawbacks of passive control is that the feeling of control can diminish when the cognitive tasks used in active control are removed.

One of the most exciting areas of detecting subconscious brain states is the field of interpreting emotional states within EEG. Although still a relatively new area of research previous studies have demonstrated the suitability of EEG in attempting to detect both a user's emotional (often referred to as affective) state [12] as well their emotional response to music.[13] One system was piloted (see section 4.1) that provides automated music selection by means of emotion detection from EEG. The results indicate that EEG can reliably be used to determine a listener's affective state and control a music selection and playback system with this information in real time. Music determined by emotions appears to be a natural step forward due to the emotional associations inherent with music for many listeners. This link is exemplified by recent studies on affective algorithmic music composition systems [14] alongside research that indicates a strong link between the use of emotionally charged music and improvements in cognitive performance.[15] However, studies where emotion recognition in a BCI is utilized can be difficult to quantify if success is measured against intention. This challenge is also increased as responses to music can often be unpredictable. These factors highlight a need for experimentation with real-time systems for rapid feedback against a set of carefully selected stimuli.

To explore this hypothesis away from the laboratory environment and in a more musical setting we present a multi-brain affective-BCMI (further referred to as the $a$-BCMI), for live recital of the piece The Space Between Us. The Space Between Us presents an artistic step towards how brainwaves may be harnessed to promote shared, affective experiences between performer and audience. The approach used to mapping measures of EEG to music provides a platform for designing performance systems that respond in real time to the affective states of participants. The objective of the piece is twofold; to determine whether affective measures in EEG can be influenced by music in an $a$-BCMI system, and to provide an artistic interpretation of the emotional trajectories of participants in a live performance setting. A particular area of interest is the nature of interaction between users and whether such a system can adapt to their affective responses. The $a$-BCMI system monitors the affective states of one performer and one audience member and uses this to select sections of a pre-composed musical score, which is then performed with deliberate emotional connotations as specified by the $a$-BCMI readings. The multi-brain system provides a novel approach to reflect the user's affective state through music using music as a stimulus to further influence users' states as part of a live performance. The system aims to move the affective states of two users closer together, creating a shared emotional experience through the music that is based on the emotional measures extracted from the EEG.

\subsection{Defining emotion in music}

Music psychology typically documents three types of emotional responses to music: emotion, affect and mood.[16] These can be considered as the reaction to sudden changes of musical expression, the perception and induced feeling of the emotional tone of music, and a longer-lasting emotional association that can be revisited with memory.[17] Scherer proposes a design-feature model in an attempt to differentiate between emotions and feelings, defining utilitarian emotions as relatively brief periods of synchronized response to the evaluation of an external or internal event, both liable to rapid change and highly susceptible to musical elicitation.[18]

Individuality presents an unpredictable and important influence on a listener's emotional response to music. There is a need for experimentation with real-time systems using rapid feedback against a set of carefully selected stimuli to develop systems that are tailored to the individual. Responses will differ from person to person depending on a range of factors such as cultural and social interpretations, preferences, prior experience, memory and so forth. An individual's emotional response to the same piece of music may vary according to factors such as the time of day, fatigue or other dynamic variables. Additionally, it has been recognized that in the field of music and emotion investigations with social approaches (research involving interactions and shared experiences) are receiving less attention than research based on solo interactions with music.[17]

Russell's circumplex model of affect [19] provides a way of parameterizing emotional responses to musical stimuli in two dimensions: valence (positivity) and arousal (energy or activation), as shown in Figure 1. This model can be mapped together with Hevner's adjective cycle [20] to create a dimensional-categorical model that 


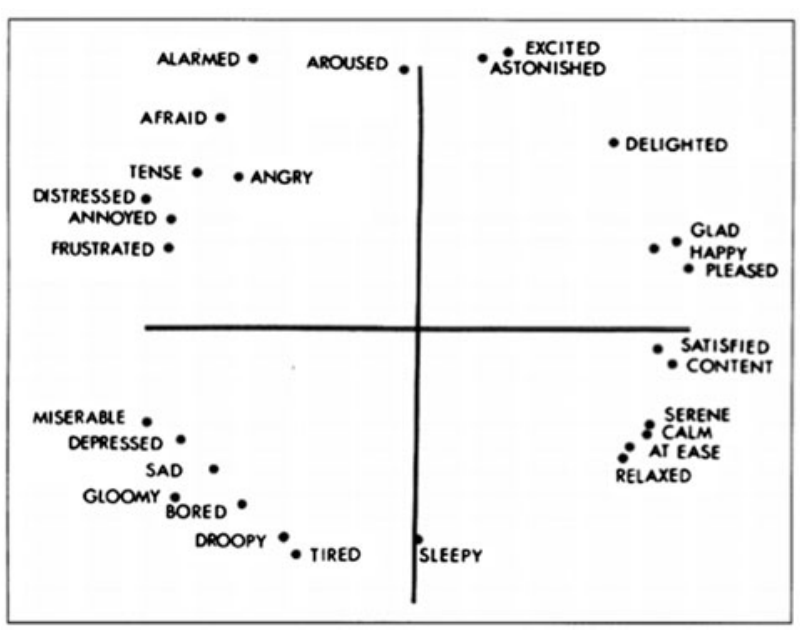

Figure 1. Circumplex model of affect, from Russell [19, p.1168]. Adjectives have been scaled in two dimensions, with valence on the horizontal axis and arousal on the vertical axis.

has been widely corroborated by other studies of music and emotion across two dimensions.[21,22] Furthermore the $2 \mathrm{D}$ model is well documented with respect to music in terms of neurophysiological measurement by means of EEG.[23-25] Russell's model ties in well with Scherer's definitions of utilitarian emotions, which are responses (not reactions) to music that include anger, sadness, happiness, fear, excitement and desperation. These responses can be considered as brief moods of high emotional intensity that are susceptible to change from stimuli. It is the effect of music on these moods that we wish to explore, and an approach is taken to compose musical sequences that reflect the emotional connotations of an affective state to induce such moods and enforce them over the duration of a sequence. In the system we present EEG is measured across the final periods of musical sequences to determine whether this has been achieved.

For the $a$-BCMI system we divide the circumplex model into quadrants which are then indexed via a Cartesian co-ordinate such that 12 discrete co-ordinates can be referenced corresponding to individual affective states across a range of arousal $(a-a 6)$ and with positive or negative valence $(v-1$ or $v 1)$, as shown in Figure 2 . Two axes give four quadrants, each of which is subdivided by three to give 12 affective states. Twelve adjectives were selected from the circumplex model such that 'basic' emotions (sad, calm, angry, and happy) are positioned such that adjectives for lower and higher arousal levels can be spaced as evenly as possible from these basic descriptors. In this manner, a co-ordinate of $(v-1, a 1)$ would refer to tired. Two adjectives from Hevner's list were deliberately avoided in the selection process, sleepy and aroused, as they were both placed near to the center of the circumplex model of affect (shown in Figure 1) and might therefore be considered ambigu-

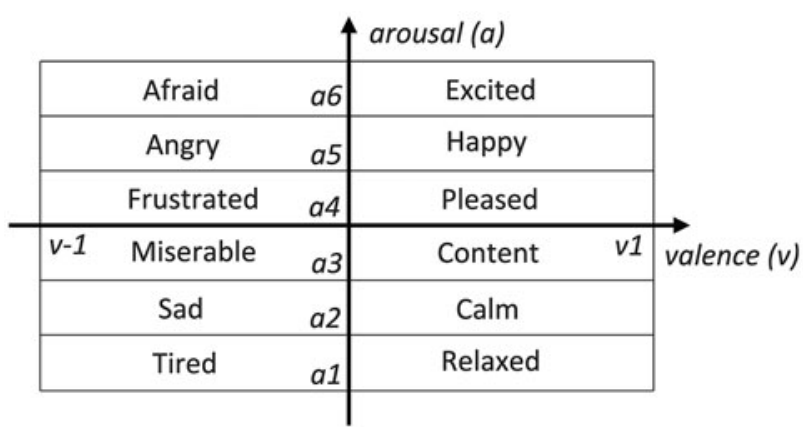

Figure 2. Values of affective states. Quadrants with 12 discrete affective adjectives from the circumplex model (afraid, angry, frustrated, excited, happy, pleased, tired, sad, miserable, relaxed, calm and content).

ous as to their valence despite a clear differentiation in arousal level between the two. There is no reason why additional adjectives could not be incorporated in the future in this model, providing they are scaled appropriately from a categorical model.

The main emotions (sad, calm, angry, and happy) can all be seen in the second and fifth levels $(v-1, a 2)$, $(v 1, a 2),(v-1, a 5)$ and $(v 1, a 5)$ respectively. An emotional trajectory moving from pleased via happy to excited can be represented by a vector which gradually increases in arousal whilst maintaining positive valence: $(v 1, a 4),(v 1, a 5),(v 1, a 6)$.

\section{Perceived vs induced emotions}

When designing affective-led systems it is important to recognize the distinction between the perceived emotions of a piece of music (i.e. when a listener reports that it 'sounds happy') and music's ability to induce an emotional state within a listener (i.e. when a listener reports that it makes them 'feel happy'). These two states are not necessarily linked by the same emotion and are not necessarily interdependent; one can exist without the other. For example a listener may understand the sad tone of a composition, but remain entirely unmoved by it. In the same manner it is not uncommon for a piece of music to evoke strong emotions in one person, whilst leaving another person feeling cold, even though both listeners may be able to translate the intended emotion of the composition. A further phenomenon associated with music listening is the way listeners use music with a strong emotional association to reinforce positive moods of an opposite affective state. This is particularly apparent in studies that monitor the pleasing effects of listening to sad music, which has been shown to improve mood.[26,27] This notion of mood enhancement is also acknowledged in studies that highlight the importance of affect-matching music to mood, to help improve 
cognitive performance.[15] Although improving cognitive performance is not a primary concern within our research this idea of affect-matching presents an ideal gateway towards engaging or locking-in the listener's affective mood with music before altering their state through the use of an affective trajectory.

An $a$-BCMI system relies on the ability of music to induce an associated affective state. As affective states in response to music are dynamic in nature (as discussed above, they may differ depending on abstract circumstances and may change significantly over time), a system must adapt to the listener accordingly in order to be successful. Therefore the $a$-BCMI we present is designed to match the mood of a user and then update the music being played in response to changes in the mood of the user. This is the approach undertaken in the Affective Jukebox. For The Space Between Us the system monitors whether a user can subconsciously affect-match themselves to the changes in music related to trajectories across the model in Figure 2. An $a$-BCMI that applies neuro-feedback to manipulate the user's affective states during performance provides an interesting model for designing specific emotionally targeted performances. Furthermore, a live performance setting provides a visual realm for communicating emotions whereas monitoring EEG responses provides a separate measure as to whether the intended affective state is actually being induced by either the performer, the listener or both.

\section{Measuring affective states from EEG}

There have been a number of methods reported to measure levels of arousal and valence within EEG, but as yet there is no standardized set of measures.[17] One common approach to determine positivity within a state of mind is to measure levels of theta and alpha bands across the scalp to determine brain synchronicity. Aftanas and Golocheikine purported that this symmetry across the hemispheres of the brain, observed during meditation, is associated with positive emotions and can be used to provide a scale for valence.[28] In 2001 Schmidt and Trainor proposed a means of categorizing emotional responses to music in EEG through measuring levels of arousal and valence in the alpha band $(8-12 \mathrm{~Hz})$ via electrodes placed on the anterior area of the forehead over the frontal lobe. Here the level of arousal correlates to the spectral power of the band.[24] Their experiments indicated that during active listening (attentive focusing or feeling the music), music with known emotional qualities can induce predictive EEG patterns. In 2010 Lin et al. monitored levels across four bands, delta $(1-3 \mathrm{~Hz})$, theta $(4-7 \mathrm{~Hz})$, alpha and gamma $(31-50 \mathrm{~Hz})$ to discern relative levels of emotion recorded in response to music listening between self-reported emotions from subjects.[13] In 2012 Ramirez and Vamvakousis proposed a method for detecting affective states in response to musical stimulus. During experimentation, they measured levels of arousal and valence across a series of subjects during music listening.[23] Stimulus were selected from the IADS (International Affective Digitized Sounds) library,[21] which contains emotion-annotated sounds, and their results indicated that the method was capable of accurately detecting the corresponding affective response between a range of $77 \%$ and $88 \%$. Their method is both simple to implement and showed potential for use in a real-time BCMI. In a later implementation of the same method Giraldo and Ramirez showcase a BCMI that maps measures of arousal and valence to a MIDI instrument in real time.[29] Here, their system is able to musify measures of affect and reflects a musical representation of affect to a user through real-time neurofeedback. The two BCMIs we present in this article also adopt Ramirez and Vamvakousis's method. The first BCMI documented here, the Affective Jukebox, aims to validate the method for use in a real-time BCMI for music selection. Here, affective measures are used to determine the continuous selection of music with corresponding emotional ratings. A user study with the Affective Jukebox, discussed in section 4.1, indicates that the method can be successfully employed for selecting affective-led music, and this informs the use of the method in the design for The Space Between Us, where pre-composed music corresponding to affective states across the two-dimensional space is selected during a live performance. The Space Between Us is designed to measure levels of arousal and valence within two subjects' EEG and selects sections of the pre-composed music in response to their affective measures.

\section{Method}

To measure brainwaves unobtrusively in a live performance environment a minimal number of electrodes is desirable. In our system EEG is measured with electrodes placed across the prefrontal cortex using the international 10/20 standard, across positions $\mathrm{AF}, \mathrm{F} 3$ and $\mathrm{AF} 4, \mathrm{~F} 4$ with reference electrode at position $\mathrm{Cz}$ and ground electrode at FPz. To determine levels of arousal we measure the ratio of alpha band power, which is inversely proportional to demanding cognitive tasks, and beta band power, which is associated with increased alertness and cognitive processing,[30] and has also been linked in other studies to an increase in arousal, separate from valence, $[31,32]$ as shown in equation 1 . We measure valence as the balance of overall power in both bands across the left and right hemispheres, shown in equation 2 , in order to indicate a difference between a motivated approach and a more negative, withdrawn mental state. 


$$
\begin{gathered}
\frac{(\beta A F 4+\beta F 4)}{(\alpha A F 4+\alpha F 4)}-\frac{(\beta A F 3+\beta F 3)}{(\alpha F 3+F 3)} \\
\frac{\beta \mathrm{AF} 4}{\alpha A F 4}-\frac{\beta \mathrm{AF} 3}{\alpha A F 3}
\end{gathered}
$$

EEG is recorded into Matlab using the g.tec Real-Time Analysis toolkit. Data are pre-processed using a $50 \mathrm{~Hz}$ notch filter to reduce line noise, and artifacts caused by muscle movement or electrical interference are removed by segmenting incoming EEG into epochs of samples (50\% overlap; Hanning window) and rejecting those that are clipped above a threshold of $+100 \mu \mathrm{V}$. Alpha- and beta-band power is extracted by applying fifth-order bandpass filters whereby each sample is squared and averaged over consecutive samples with a window length of 128 samples. We are interested in measuring the affective state in response to music currently being listened to. As such, values of spectral power are normalized across a window of a pre-specified duration, in this case the final $20 \mathrm{~s}$ of a $90 \mathrm{~s}$ window, though different compositions might require different window sizes depending on the piece. This allows time for the listener to familiarize with the music and then settle towards an overall affective response. This method is useful to counter the known effect of diminishing arousal over time as subjects familiarize themselves with the stimuli and the environment.[33] A threshold-base classifier is trained to adapt to individual user responses during a calibration phase that is undertaken at the beginning of every interaction with the system. The calibration phase measures responses at the outer limits of the model against the musical stimuli. The system contains an array of musical sequences, each with an intended arousal and valence value. A musical sequence is selected by a transformation algorithm, which maps a user's EEG arousal and valence measure to a corresponding musical sequence or to a set of sequences, across the $2 \mathrm{D}$ space. The process of EEG measurement through to music selection is illustrated in Figure 3. At the end of each window the affective state is approximated and used by the system to determine the next musical selection, or recorded for comparison against the system trajectory selection.

\subsection{The Affective Jukebox: confirming EEG measures with user self-assessment}

A previous study was conducted to explore the hypothesis of whether arousal and valence could successfully be detected in real time using the passive method outlined above. Participants were asked to actively focus on how the music made them feel and success was determined by comparing EEG data against user self-reports. Akin to the control one has over song selection with a traditional music jukebox, the BCMI system selects musical

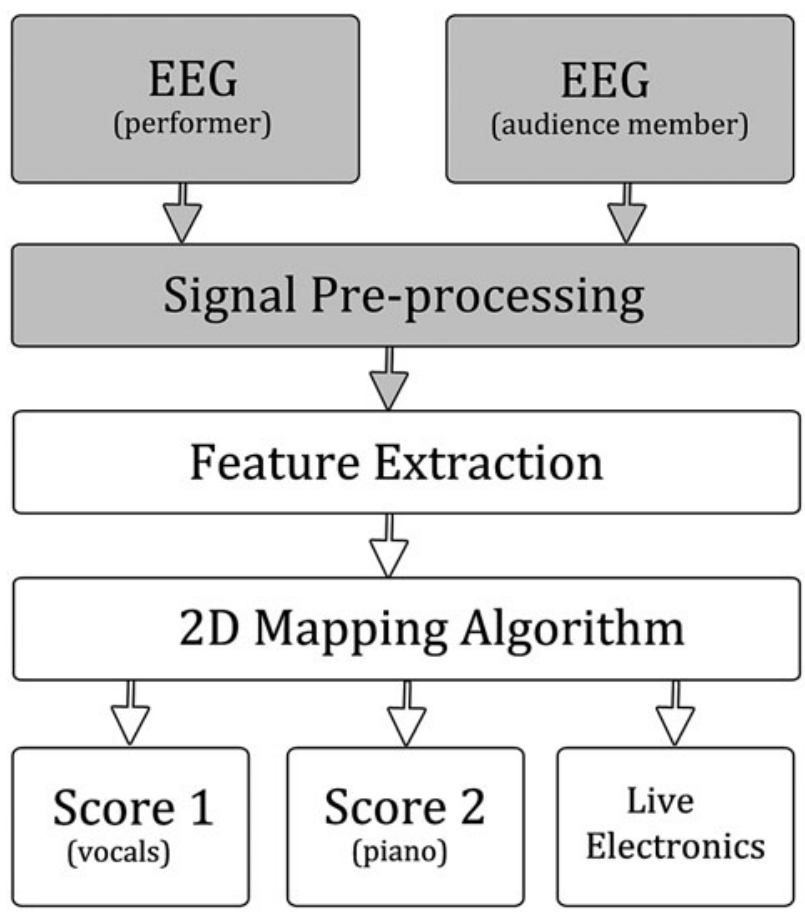

Figure 3. Signal flow within the $a$-BCMI. The 2D mapping algorithm selects musical sequences, which are presented as a score, depending on different rules during performance. The system either selects a target state and corresponding score to match the arousal and valence of a user or it determines a target state by calculating the mean values of both users' arousal measures.

clips based on a user's mood. Clips with suggested emotional qualities taken from crowd-sourced metatagging were used in the study. Each clip is played back to a user over loudspeakers, generating a constant playback of musical excerpts in response to the affective state measured during the previous excerpt.

Results of this pilot study indicated that even with a limited number of listeners, mean agreement was relatively high $(85 \%)$ with a statistically significant standard deviation $(\sigma=0.14)$, suggesting that there was a good degree of correlation between the EEG measurement and the mood meta-tagging that was used to select the stimuli. An interesting observation was that outliers existed where users indicated that their perception of stimuli was not congruent with the associated tags used in the experiment, strengthening the need for individualized stimuli in applications of this kind. A separate observation of note is that when music was successfully matched to mood the same state was detected for a number of iterations of the paradigm. On reflection this appears to follow common sense, as when music reflects a mood that is pleasing there is little motivation for mood to suddenly change. The fact that this state did change after a short number of iterations is probably due to the fact that only 
one stimulus was selected per mood, and users responded negatively to on-going repetitions. A separate report, including detailed analysis of the paradigm and these results, is available in [9].

\section{The Space Between Us}

To recap, the piece is designed to explore two main enquiries: firstly, whether affective measurements in EEG respond to pre-composed music in both a performer and a listener during live performance, determining whether plotting affective trajectories across the model in Figure 2 can influence the detected affective states induced during the performance in a neuro-feedback-derived process. The second is to monitor the affective interactions between two users during music-making to help determine whether future BCMI systems can be built to adapt to this interaction in the future. Collaboration between people is a highly regarded feature of musical participation and an affective channel of communication that adapts to users offers an exciting prospect in designing BCMI systems for a variety of uses, outside performance. One example is in BCMI systems for interaction between therapist and patient.

The piece is composed for grand piano, live electronics (controlled by the pianist) and voice. Before the performance begins the singer and an audience member are fitted with brain caps, with electrodes configured in the manner described in section 4, to measure EEG simultaneously throughout the performance. The system records their affective state at the end of each window, as outlined above, and maps this to discrete pre-composed musical sequences (essentially, small chunks of score), which are then in turn performed. The system is synchronized to a global clock that acts both as a loose visual metronome and to trigger the event of a new score selection at the end of each window $(1$ window $=90 \mathrm{~s})$. The clock is presented as a counter alongside the score to the singer and the pianist on-stage. Depending on the measured arousal valence response of the two subjects, a variety of affective trajectories can be plotted across the 2D grid (Figure 2). The system displays the corresponding score whilst monitoring changes in arousal and valence at the end of each window. EEG data are also written to a text file for off-line analysis post-performance.

\subsection{Structure}

The music of the piece contains 12 pre-composed musical sections. Each section is composed using specific musical features with affective correlations as emotional cues. Essentially it is the sequencing of the sections that is led by the affective states, with the intention that the final section of each window will subsequently influence the affective state as measured from the performer and the audience member at the end of the current section. For the final $20 \mathrm{~s}$ of each window both the performer and the audience member are instructed to remain physically still, to reduce interference in measuring EEG, and reflect on the mood that the current music and performance has instilled in them. A prompt is provided to the singer on screen using prominent color changes that both warns the viewer when this process occurs and displays the timing of this period.

The piece, although performed continuously, comprises three movements as shown in Figure 4 (following a randomly selected preliminary window). Each movement is four windows long and is designed to elicit three separate things. The first movement attempts firstly to affect-match the mood of the singer by selecting a score

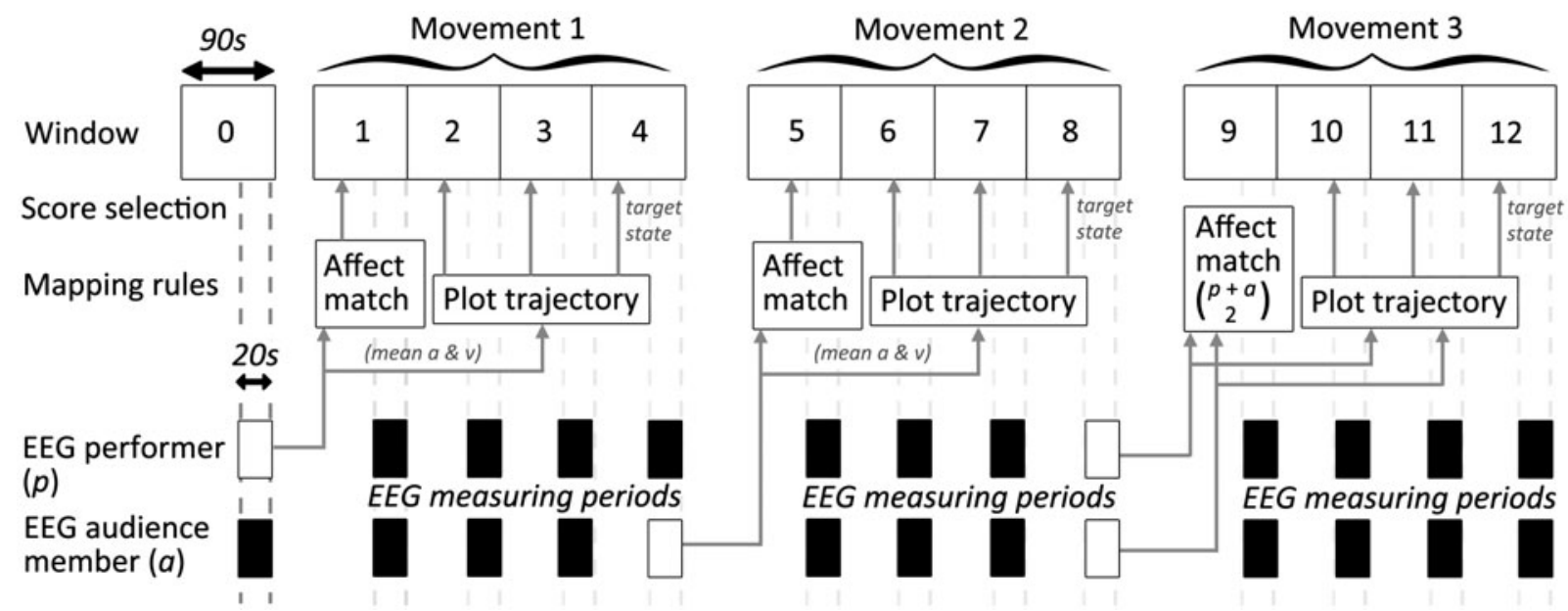

Figure 4. Structure of The Space Between Us. EEG measuring periods occur during the last $20 \mathrm{~s}$ of each window. The measures of mean arousal and valence taken indicated by white boxes are used by the rules of the mapping algorithm to select score sections and to plot trajectories. 
from their response to the randomly selected musical score of the preliminary window, and then to shift their affective state across three adjacent affective states. The second movement performs the same task for the audience member using their response to the last window of movement one for initial affect-matching, and the third movement selects the first window based on a median average of both subjects' affective states and plots a trajectory in an attempt to move both their states simultaneously and towards each other across the 2D space. This overall success of these aims is evaluated against EEG data and discussed further in section 6 .

The score for movement 1 , window 0 is selected at random and the resulting $2 \mathrm{D}$ coordinate $(\mathrm{x}=v, \mathrm{y}=a)$ recorded at the end of the window is saved as the initial state. The corresponding score of this state is selected for window 1. A target coordinate is determined by randomly selecting a state that is three steps away across the plane, either $( \pm 1 v, \pm 2 a)$ or $( \pm 3 a)$. Multiplying the target co-ordinate by $0.33,0.66$ and 1 respectively sets a trajectory across the next three windows. Associated states are selected that span the projected path and select the corresponding musical phrase from the array. Movement 2 then follows a similar procedure; however, the system takes into account the affective response of only the audience member. The mapping for movement 3 uses the coordinates of each subject from the fourth window of movement 2 as initial values, $p$ (performer) and $a$ (audience member). The difference between each individual's emotional sate, as shown in equation 1 , becomes the target value for movement 3's fourth window, again with multiplication factors for the preceding three windows, plotting a trajectory. The final window in movement 3 selects a target value of positive valence, at the closest state to the difference between $p$ and $a$ to induce a positive emotional ending to the performance experience.

$$
\sum \frac{p+a}{2}
$$

Whereas the first and second movements aim to affectmatch and then shift the mood of the singer and audience member respectively, the third movement aspires to shift the mood of both parties across the same trajectory, together. Although EEG is being measured from both users throughout the entire performance the intention was to design and test a mapping system where the participants did not feel pressured or need to take responsibility for the outcome of the system.

It was necessary to determine trajectory paths in advance so that both performers could be warned of the upcoming score for many of the windows in advance. In the same way that musicians read ahead when sight-reading this helps prepare for changes and allows for smooth transitions between windows, and as such this is an implementation challenge which may be unique to the field of real-time BCMI. Additionally, the effect of repetitive score selection due to an affect-matching feedback loop, as observed in the Affective Jukebox experiment outlined above, can be minimized by this approach.

An illustrative video of a performance and rehearsal session of the premiere in Berlin, Germany, December 2014, can be viewed online here: http://www.vimeo.com/ $116,013,316$.

\subsection{System design}

The multi-user $a$-BCMI system is built around two EEG systems with identical components that include brain caps, electrodes, hardware and software. Equipment was selected to be portable and facilitate comfortable participation. A wireless EEG setup was used that allowed for physical separation from the PCs used to receive and process EEG data, a practical necessity in the live performance environment. The performer was required to move freely during periods when EEG was not being detected, and it was important for the participating audience member to feel as though they were an extension of the system, without feeling distracted or overwhelmed by the equipment in their vicinity.

Two laptop PCs are used on-stage to allow both performers to see the scores of their respective parts, as illustrated in Figure 5. A third laptop PC is situated offstage next to the audience member. A closed wireless local area network (WLAN) allowed for data transfer across all three PCs for EEG processing, score selection, and clock synchronization.

\subsubsection{System components}

- Singer and audience member brain caps

EEG signals are measured with active g.tec Sahara electrodes, a g.tec Sahara amplifier and a gMOBILAB+digitizer. Data are sent via Bluetooth to PC1 and PC2 for the performer and audience member respectively. Active electrodes require no gel application and are therefore more straightforward and quicker to set up than wetware systems. The active g.tec Sahara electrodes each house eight pins designed to penetrate through hair and achieve a good connection with the scalp, further reducing system impedance.

\section{- Three laptop PCs}

There are three interconnected PCs in the system, each designed to perform a different role related to its primary user. PC1 is positioned on-stage in front of the singer and placed on a music stand. The computer 


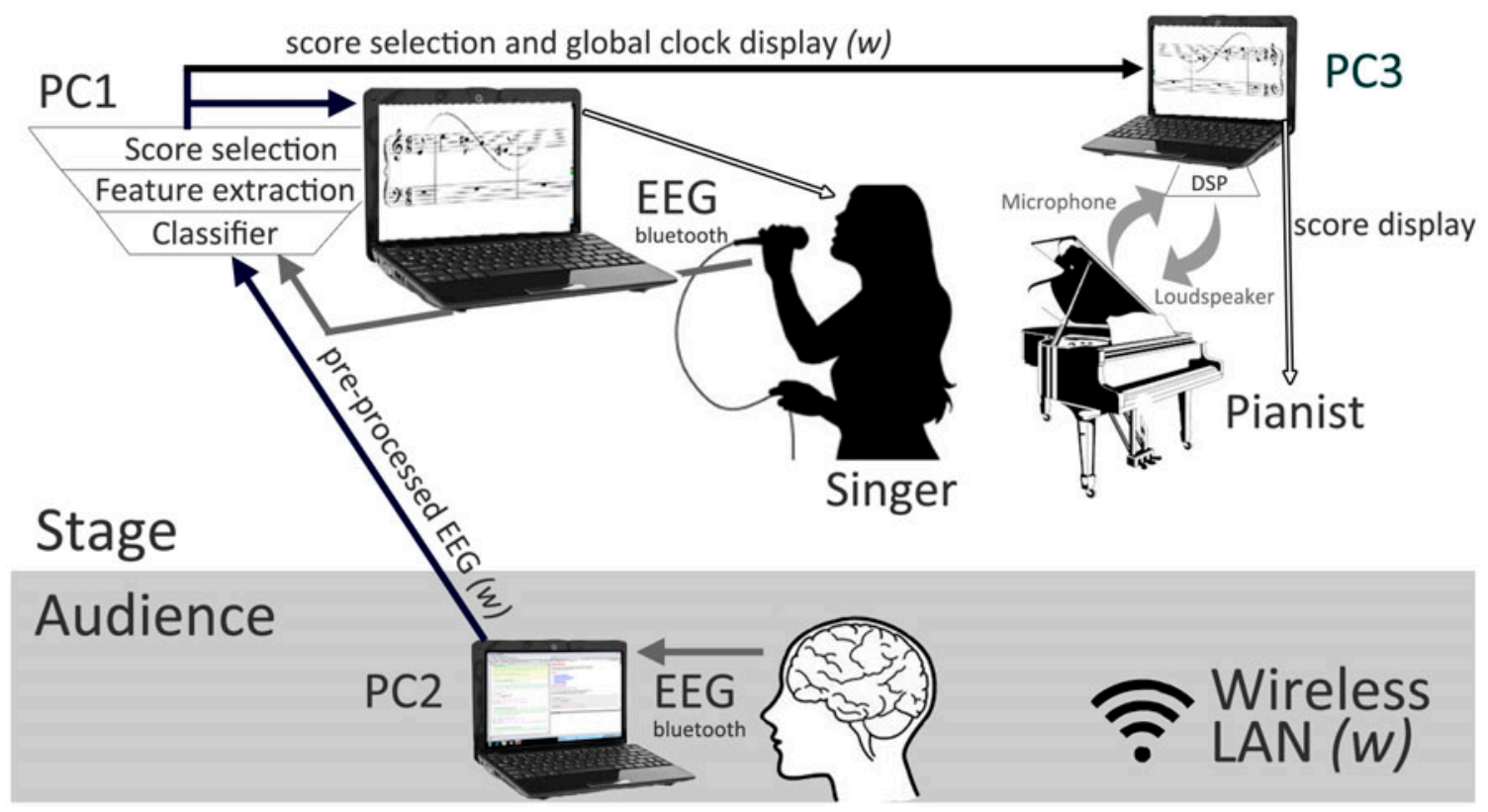

Figure 5. Signal-flow, connectivity and the layout of the main components in the multi-brain $a$-BCMI system used in The Space Between Us.

firstly pre-processes raw EEG data from the singer's brain cap in Matlab Simulink. This combines with the audience member's pre-processed EEG data wirelessly received from PC2. EEG data classification and feature extraction for both users are performed by Simulink running on $\mathrm{PC} 1$ then the scaled output values are sent to a mapping algorithm in Pure Data for score selection from Simulink via a bespoke $s$-function that converts data into the Open Sound Control (OSC) protocol to be sent internally via the User Datagram Protocol UDP. The master Pure Data patch on PC1 manages the global clock, maps affective features to the score selection and, through the visual extension GEM, displays the score for the singer.

PC2 receives raw EEG from the audience member's brain cap. Again, EEG is captured by Simulink and sent wirelessly via OSC to PC1. Global controls are housed in a third Pure Data patch to allow an engineer to start (and also to stop and reset if problems arise) the performance at will. The global commands are sent wirelessly, again via OSC, and received by the master patch in PC1.

PC3 performs two functions, to display the score and the clock to the pianist and to handle the real-time audio digital signal processing (DSP) of the piano, outlined under the bullet point below. The first function is achieved by a third Pure Data patch that receives clock updates and score selection commands, wirelessly from the master patch in PC1. These commands are passed to a GEM window in PC3, to display the score and clock to the pianist. The second function is realized through a real-time audio processing feedback loop.

\section{- Processed piano feedback loop}

A condenser microphone captures the sound from inside the body of the piano, which is fed, using an external soundcard, into a MAX/MSP patch hosted on PC3. The bespoke patch feeds the audio through a series of effects processors: spectral freeze, sample and hold, and a delay line; and then it passes it back out via the soundcard to a loudspeaker placed underneath the body of the grand piano, facing upwards so as to resonate the strings. The pianist, using the faders and buttons of a USB digital control surface connected to PC3, manually controls the parameters of the audio effects, mixing them together in real time according to instructions marked in the score. The result produces sustained ethereal characteristics that provide a subtle blend with the acoustic sound of the piano when fed back into the resonant body of the instrument. The effects are used predominantly for music phrases with negative valence and/or low arousal, where there is minimal rhythmic activity, atonal harmonic structures and drone-like sustain, specifically the states relaxed, frustrated, and angry.

\subsection{Musical composition}

The piece is composed to attempt to trigger physiological changes in EEG on an unconscious affective level, 
and also to clearly communicate the intended emotions through the music, lyrical content and the delivery of the performance. Ultimately if an audience struggles with interpreting the emotional cues in the music, then the other two aspects are designed to help aid this emotional communication. When previous studies have investigated EEG emotional correlates in response to music the focus has often been more biased towards measuring success solely within EEG data, rather than on the suitability of musical stimuli.[34] Also little has been done to study the effects of musical performance on affective correlates in EEG, though the affective potential of multimodal stimuli has been well documented.[35,36] As such, factors such as stage demeanor, delivery and lyrical content were considered useful in order to help explore this area and investigate their combined effects. To assist with this, the lyrical content for the piece was edited from copyright-free works of Percy Bysshe Shelley, an English romantic poet well regarded for the strong emotions his writing evokes.

Two of the most widely accepted musical parameters that influence arousal and valence are, respectively, tempo and mode.[33,37] Franco et al. [15] identify further expressive cues in music related to mood, such as Happy, harmonic consonance and off-beat accentuation, and Angry, harmonic dissonance and a greater density of note onsets. Additionally, the KTH performance rules provide a set of relative values for arousal and valence linked to musical performance attributes.[22] However, as the authors also recognize, it is extremely difficult to compose music according to rules based on 30-40 specific musical parameters, especially when trying to impart a coherent musical style. It was also felt important that the composition of The Space Between Us should feel intrinsically human, therefore computer-aided composition techniques, which could have abided by such strict rule-sets, were avoided. With this in mind the KTH rules were used as a guide to help apply emotional characteristics with a particular focus on global functions such as mode, tonality, rhythmic density, intensity and dynamics, and with attention paid to attributes of shorter durations such as articulation, phrase arch and punctuation, which contain ranges with particular emotionally expressive qualities.

Twelve parts to the piece were composed in-line with the 1 states categorized in the affective model. Global musical features were mapped across the axes of the two dimensions depending on their affective association. For example, rhythmic intensity increased from Tired/ Relaxed to Afraid/Excited, according to an emotional interpretation as opposed to a strict mathematical scaling. It was felt that applying a straightforward major/minor mode distinction between all positive and negative states of valence would provide too much obvious contrast and restrict the interest and overall unity of the piece, so an atonal style (music of no fixed central tonality) was used in a number of negative valence states, which provided a useful range of dissonance. Furthermore, extended and experimental performance techniques were utilized to reinforce stark changes in emotion, as well as the piano feedback digital effects routines. For example, certain parts required the piano strings to be played aggressively inside the body using a plectrum (Frustrated, Angry), and some parts required a strongly affected vocal style to help convey different intensities of emotion (Miserable, Frustrated). The composition was developed through workshop sessions with the singer, in order to cultivate an individualized stimulus set that they could connect with and feel comfortable delivering, according to the required emotional expressivity.

It would not be practical to provide the full score here; however, a brief look at the musical score from two parts provides an insight into some elements of the compositional design. Figure 6 shows the score for Tired, which is associated with minimal arousal and negative valence. The musical term calando instructs performers that the pace slows down throughout, whilst simultaneously quieting, an effect designed to mimic the notion of falling asleep. To reinforce this the piano part is a series of descending cadences constructed on a series of atonal harmonic transitions and their variations. This is then matched by a short vocal line which comes in after the piano, once the mood has been set. Both performers are instructed to perform the part with a decreasing energy which is to be perceived physically as well as aurally. The lyrics here reflect the evocation of a memory, which is often the case during the period of tiredness before sleep.

Figure 7 presents the first section of the score for Excited, which is at the opposite end of the affective model from Tired, with maximal arousal and positive valence. The score illustrates a fast tempo in 4/4 timing with a repetitive, regular piano-led beat and high rhythmic density. The notes are played in an almost staccato style, short and sharp, a technique mirrored by the vocal part to express fast bursts of energy. In the latter stages of this section dynamic changes are introduced alongside different rhythmic patterns (triplets and then 7/4) to maintain listener engagement with high arousal without relying on excessive repetition.

The lyrics for Excited are adapted from Shelley's stanzas The Cloud, a playful poem about the neverending cycle of life. The poem is written from the perspective of an electrically charged cloud eliciting strong, colorful and exciting visual imagery. Three parts were selected not to contain a vocal line, in order to present the associated state and to provide some variation within the performance. Relaxed, Happy and Afraid contained only a piano line and/or electronic feedback. 


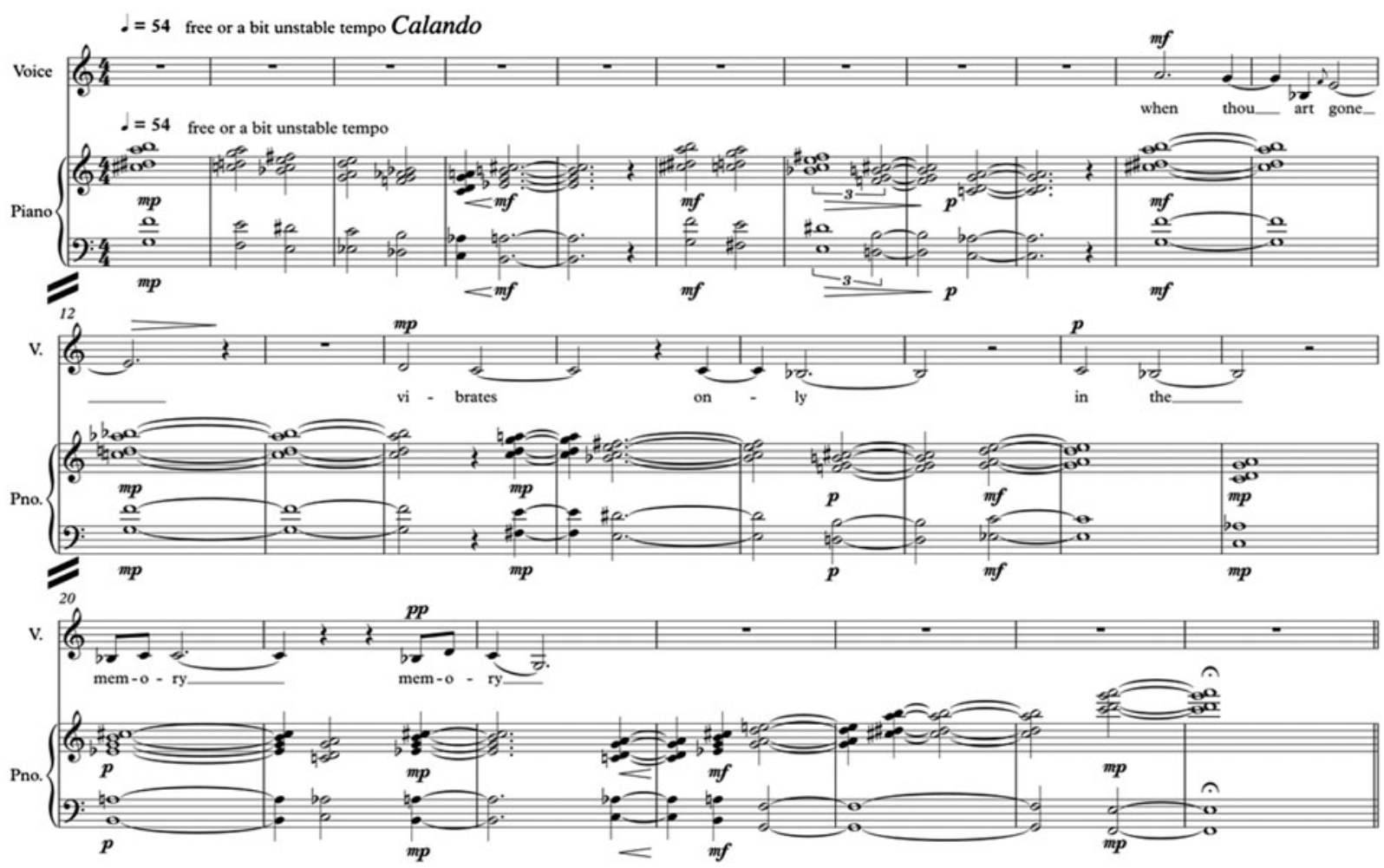

Figure 6. The vocal and piano score for Tired. The piano part contains a series of descending atonal harmonic transitions. The vocal line descends in pitch with the piano over a series of long, sustained notes as both parts slow down irregularly in tempo.

\section{Performance and observations}

Data collected during the premier performance of The Space Between Us, shown in Table 1, provide an interesting account of the measured arousal and valence in the EEG of the performer and audience participant. Statistical significance would require multiple iterations of the performance or a larger number of $a$-BCMI users in one performance. This presents two problems. Firstly, the practical demands, including the costs, of hosting either of these scenarios is outside the feasibility of our resources. Secondly, we predict that there would be large variations in data between events, even with the same users, based on the commonplace variation in affective states discussed in section 1. Measures of affective states are not definitive and it would appear logical to expect that the responses from both individuals are likely to change depending on a near infinite range of external factors that might influence their mood, resulting in a different global arrangement of the music sections upon every performance. It is therefore important to reiterate that this system and the associated performance were designed specifically for a singular experience for the participants. As such the data presented in this section are merely indicative of the potential for a multi-user $a$-BCMI system and should be read with this in mind.
Table 1 shows arousal and valence from both users, alongside the selected scores and the system's affective trajectories. Useful comparisons can be made with the arousal/valence trajectories against the musical trajectories. At the beginning of each movement the system attempts to affect-match the music (in windows 1,5 and 9) to the corresponding user's response to the music of the previous window (window $1=$ user 1 , window $5=$ user 2 , window $3=$ mean of user 1 and user 2). The mapping algorithm in the master Pure Data patch plots a global affective trajectory and the responses of both users are recorded. The results indicate a strong link with the musical trajectories and changes in user arousal. Changes in valence are noted as the difference between negative and positive values whereas ranges of arousal are scaled according to a calibration task undertaken during a rehearsal prior to performance.

The affect-matching of the system at the beginning of each window shows a clear pattern of user's arousal level closely matching the music of the previous window. Movement 1 begins with $\mathrm{Sad}$, which is taken from the affective response of the singer at the end of Tired. Sad and Tired sit next to each other in the 2D model. Similarly at the end of movement 1 user 2's response selects Afraid, which is close to the previous window's Angry music. Finally the system takes an approximate 


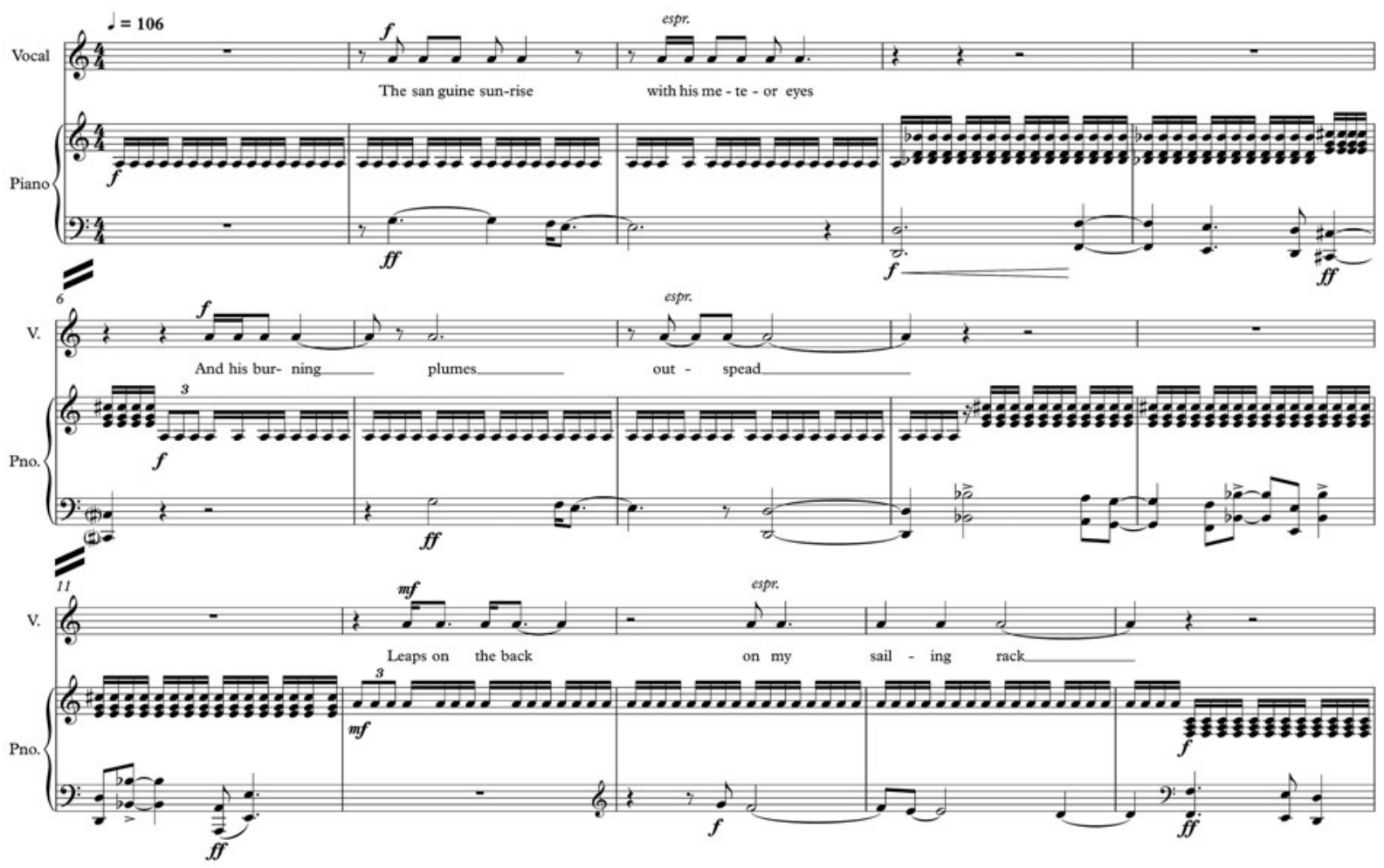

Figure 7. The first half of the vocal and piano score for Excited. The piano part leads with a regular $4 / 4$ rhythm with minimal harmonic variation. The vocal part sings the lyrics to the same pitch with a mixture of staccato and the occasional sustained note. The tempo is much faster than the score for Tired for a more energetic delivery.

Table 1. System trajectory and score selection and user EEG data. User 1 is the singer and User 2 is the audience member. The correlation between musical stimuli and affective measures from EEG can be seen in the changes across each user's affective measures (User 1 and User 2 trajectory columns) against the trajectories of the music (column heading 'Changes in score AV'). Increments (inc) and decrements (dec) are used for labeling as the system is calibrated slightly differently to the response of each user.

\begin{tabular}{|c|c|c|c|c|c|c|c|c|c|}
\hline Window & $\begin{array}{l}\text { Affect-matched } \\
\text { state }\end{array}$ & $\begin{array}{l}\text { System trajectory } \\
(\text { bold = target state })\end{array}$ & $\begin{array}{l}\text { Change in score } \\
\mathrm{AV} \text { measures }\end{array}$ & $\begin{array}{l}\text { User } 1 \\
\text { arousal }\end{array}$ & $\begin{array}{l}\text { User } 1 \\
\text { valence }\end{array}$ & $\begin{array}{l}\text { User } 1 \\
\text { trajectory }\end{array}$ & $\begin{array}{l}\text { User } 2 \\
\text { arousal }\end{array}$ & $\begin{array}{l}\text { User } 2 \\
\text { valence }\end{array}$ & $\begin{array}{l}\text { User } 2 \\
\text { trajectory }\end{array}$ \\
\hline 0 & & $\begin{array}{l}\text { Tired (randomly } \\
\text { selected) }\end{array}$ & & 1.65 & -0.1 & & 1.54 & 0.23 & \\
\hline 1 & Sad & & $(a=$ inc $)$ & 1.85 & 0.43 & $\begin{array}{l}(a=\text { inc }) \\
(v=\text { inc })\end{array}$ & 1.59 & 0.28 & $(a=$ inc $)$ \\
\hline 2 & & Miserable & $(a=$ inc $)$ & 1.82 & 0.44 & $(a=\mathrm{dec})$ & 1.45 & -0.09 & $\begin{array}{l}(a=\mathrm{dec}) \\
(v=\mathrm{dec})\end{array}$ \\
\hline 3 & & Frustrated & $(a=$ inc $)$ & 1.86 & 0.55 & $(a=$ inc $)$ & 1.48 & 0.18 & $(a=$ inc $)$ \\
\hline 4 & & Angry & $(a=$ inc $)$ & 1.92 & 0.48 & $(a=$ inc $)$ & 1.66 & 0.11 & $(a=$ inc $)$ \\
\hline 5 & Afraid & & $(a=$ inc $)$ & 1.72 & 0.58 & $(a=\mathrm{dec})$ & 1.54 & -0.18 & $(a=\mathrm{dec})$ \\
\hline 6 & & Afraid & constant & 1.62 & 0.21 & $(a=\mathrm{dec})$ & 1.52 & 0.2 & $(a=\mathrm{dec})$ \\
\hline 7 & & Excited & $(v=$ inc $)$ & 2.23 & 0.44 & $(a=$ inc $)$ & 1.65 & 0.31 & $(a=$ inc $)$ \\
\hline 8 & & Нарру & $(a=\mathrm{dec})$ & 0.46 & 0.46 & $(a=\mathrm{dec})$ & 1.66 & 0.25 & $(a=$ inc $)$ \\
\hline 9 & $\begin{array}{l}\text { Miserable } \\
\text { (Happy) }\end{array}$ & & $\begin{array}{l}(v=\operatorname{dec}),(a= \\
\text { dec })\end{array}$ & 1.58 & 0.4 & $(a=\mathrm{dec})$ & 1.75 & 0.24 & $(a=\mathrm{dec})$ \\
\hline 10 & & Pleased & $\begin{array}{l}(a=\text { inc }),(v= \\
\text { inc })\end{array}$ & 1.77 & 0.62 & $(a=$ inc $)$ & 1.73 & 0.2 & $(a=\mathrm{dec})$ \\
\hline 11 & & Нарру & $(a=$ inc $)$ & 1.96 & 0.42 & $(a=$ inc $)$ & 1.55 & 0.19 & $(a=\mathrm{dec})$ \\
\hline 12 & & Excited & $(a=$ inc $)$ & 1.68 & 0.31 & $(a=\mathrm{dec})$ & 1.79 & 0.22 & $(a=$ inc $)$ \\
\hline
\end{tabular}


measure of both users' affective response of window 8 , Happy. The mean of the two measures pre-empts a repeat of Happy (as indicated in brackets in Table 1) but the system follows an in-built rule to avoid repetition of states and shifts the score selection by a random measure of up to two steps across the 2D space.

The data in Table 1 show that valence in both users generally stays positive throughout, and does therefore not appear to be strongly linked to changes in the music. This could be the result of either the composition lacking enough negative musical features although another explanation could be that this is an indication of the levels of mental engagement required of both the performer and the audience member in an intense concert environment. This suggests that investigations of arousal are perhaps more telling of the affective experiences of both parties. Significantly, across both users measures of arousal move upwards or downwards in the direction of the target state in each movement, even with occasional deviation. This suggests a substantial success of the system and the performance in the ability to move user arousal across a trajectory.

A number of anomalies exist in the data, which are perhaps to be expected when analyzing a performance of this kind. In particular the affective responses of the audience member have less correlation with the musical trajectories than those of the singer. In light of the fact that the composition was developed during workshop sessions with the singer (where EEG was also monitored), this might be expected, as the music became individualized to the singer during these sessions. The audience member had no previous exposure to any of the music and is perhaps likely to have less emotional attachment towards, and also less emotional understanding of the piece. Other incongruences in the data may be the result of musical factors. For example, in the first movement, window 2, the performance of Miserable induces negative changes in arousal against the previous $\mathrm{Sad}$ section. This may be indicative of the music either reinforcing negative emotions or even that Miserable feels less energized than $\mathrm{Sad}$. The sections are both quite similar in musical terms, both with a slow tempo and in an atonal key. It is interesting that this drop in arousal occurs in both users. Another interesting outcome can be seen in windows 5 and 6 , where measures of arousal drop across both users. A system error allowed Afraid to be played twice in a row during windows 5 and 6 (the rule avoiding repetition failed to avert this). Interestingly, this allows us to see the effect of prolonged music against all the other shorter sections. Here, measures of arousal decrease across both windows. This could perhaps be because of two possibilities. Firstly, there is no vocal part during Afraid. The piano attempts to use tonal relationships to convey the feeling of Afraid, but there is far less drama than in the atonal and vocally shouted
Angry, which precedes Afraid. Secondly, as we have mentioned, arousal has been known to decrease with repeated exposure to the same stimuli. Exactly the same part being performed twice may well have contributed to this. A further observation is in the role of the physical demands of performing, which are likely to play a significant role in the singer's overall levels of arousal and valence. Other bio-signal measures of arousal taken from the singer are likely to corroborate arousal measures in EEG readings as they are more closely linked to physical exertion. For example, using an ECG/EKG, a faster heart rate is likely to be caused by singing high-intensity phrases which place a greater demand on the cardiovascular system.

There are a number of limitations with active BCI control for music-making. Two in particular are particularly relevant to music-making activities. The first is the amount of time between cognitive processing and the corresponding control signal being detected, and the variability of this time. The second is a lack of simultaneous controls on offer. Both of these attributes, which are analogous (and typically related in BCMI) to real-time musical response and the musical notion of polyphony, are intrinsic to the design of interactive digital musical interfaces.[38] In response to the need for more complex control (through simultaneous dimensions) is a shift towards combining active control with secondary, passive brainwave detection methods $[39,40]$ to create hybrid-BCI control. Our motivation for investigating measures of passive control in EEG stems from this school of thought: we want to develop BCMIs that offer deeper and more complex control than active systems alone, combining passive and active methods to create novel BCMIs. However, before this can be fully realized we are keen to explore the potential of passive control alone, and the research outlined in this paper focuses on detecting passive measures related to emotions with $\mathrm{EEG}$, for control over music in a BCMI system.

\section{Conclusions}

BCI presents a useful control system for musical applications. A few systems already exist, most of which rely on active control methods mapped to musical functions. Passive BCI systems are not always limited to the restraints of active control methods and present an interesting partnership for music systems whereby mental states detected from EEG can be mapped to music generation, in particular affective BCMIs. Neuro-feedback allows for individualized music in a BCMI to be selected in response to measures of affect, which addresses the problem of intersubject differences in emotional response and how measures of affect can alter at different times.

We present a passive system which reads emotions in two dimensions. The metrics were validated in a study that 
used self-report to corroborate the EEG metrics in the Affective Jukebox. In this study, the aim was to see how two people can have a musical interaction, and whether the passive system can find some emotional common ground.

The piece The Space Between Us indicates that arousal can successfully be measured using the EEG method outlined in [23] during musical performance. Early results suggest that some interesting influence over arousal was achieved, whereas measures of valence were less conclusive. The system has constraints which are practical in order to be used in a real-world context, away from laboratory conditions. A minimal electrode set-up is used and a rudimentary approach to classifying affect in real-time was developed.

Hybrid active-passive BCMI systems present an interesting area for future studies as they present more complex combinations of user control, which could result in interesting musical applications.

\section{Acknowledgements}

The authors would like to acknowledge the support of a PhD scholarship awarded by Plymouth University. The music composition for The Space Between Us was developed in collaboration with the composer Miss Weiwei Jin.

\section{Disclosure statement}

No potential conflict of interest was reported by the authors.

\section{References}

[1] Mason SG, Birch GE. general framework for brain-computer interface design. IEEE Trans. Neural Syst. Rehabil. Eng. 2003;11(1):16.

[2] Grierson M, Kiefer C, editors. Better brain interfacing for the masses: progress in event-related potential detection using commercial brain computer interfaces. 29th International Conference on Human Factors in Computing Systems; 2011; Vancouver, Canada.

[3] Miranda ER, Magee WL, Wilson JJ, et al. Brain-Computer Music Interfacing (BCMI): from basic research to the real world of special needs. Music and Medicine. 2011;3 (3):134-140. doi:10.1177/1943862111399290.

[4] Lucier A. Statement on: music for solo performer (1971). In: Rosemboom D, editor. Biofeedback and the Arts: Results of Early Experiments. Vancouver: Aesthetic Research Center of Canada; 1976 pp. 60-61.

[5] Eaton J, Miranda E. On mapping eeg information into music. In: Miranda ER, Castet J, editors. Guide to Brain-Computer Music Interfacing. London: Springer; 2014. p. 221-254.

[6] Guger C, Edlinger G, Krausz G. Hardware/software components and applications of BCIs. In: Fazel-Rezai R, editor. Recent Advances in Brain-Computer Interface Systems. Croatia: Rjeka; 2011. p. 1-24.

[7] George L, Le' cuyer A, editors. An overview of research on "passive" brain-computer interfaces for implicit human-computer interaction. International Conference on Applied Bionics and Biomechanics ICABB 2010 2010; Venice Italy.

[8] Eaton J, Jin W, Miranda E, editors. The space between us: a live performance with musical score generated via affec- tive correlates measured in eeg of one performer and an audience member. New Interfaces for Musical Expression (NIME'14); 2014; London, UK.

[9] Eaton J, Williams D, Miranda E, editors. Affective jukebox: a confirmatory study of EEG emotional correlates in response to musical stimuli. Joint ICMC | SMC 2014 | 11th Sound and Music Conference |14th International Computer Music Conference; 2014; University of Athens, Greece.

[10] Baier G, Hermann T, Stephani U. Event-based sonification of EEG rhythms in real time. Clin. Neurophysiol. 2007;118(6):1377-1386. doi:10.1016/j.clinph.2007.01.025.

[11] Strehl U, Leins U, Goth G, et al. Self-regulation of slow cortical potentials: a new treatment for children with attention-deficit/hyperactivity disorder. Pediatrics. 2006;118(5): e1530-e1540. doi:10.1542/peds.2005-2478.

[12] Chanel G, Kronegg J, Grandjean D, et al. Emotion assessment: arousal evaluation using eeg's and peripheral physiological signals. In: Gunsel B, Jain AK, Teklap AM, Sankur B, editors. Multimedia content representation, classification and security. Springer, LNCS; 2006; 4105. p. 530-537. doi:10.1007/11848035 70 .

[13] Lin Y, Wang C, Jung T, et al. EEG-based emotion recognition in music listening. IEEE Trans. Biomed. Eng. 2010;57(7):9. doi:10.1109/TBME.2010.2048568.

[14] Williams D, Kirke A, Miranda ER, et al. Investigating affect in algorithmic composition systems. Psychol. Music. 2014. doi:10.1177/0305735614543282.

[15] Franco F, Swaine JS, Israni S, et al. Affect-matching music improves cognitive performance in adults and young children for both positive and negative emotions. Psychol. Music. 2014;42(6):869-887. doi:10.1177/ 0305735614548500.

[16] Russell JA, Barrett LF. Core affect, prototypical emotional episodes, and other things called emotion: dissecting the elephant. J. Pers. Soc. Psychol. 1999;76(5) p. 805.

[17] Lamont A, Eerola T. Music and emotion: themes and development. Musicae Scientiae. 2011;15(2):139-145. doi:10.1177/1029864911403366.

[18] Scherer KR. Which emotions can be induced by music? what are the underlying mechanisms? and how can we measure them? Journal of New Music Research. 2004;33 (3):239-251. doi:10.1080/0929821042000317822.

[19] Russell JA. A circumplex model of affect. J. Pers. Soc. Psychol. 1980;39(6) p. 1168.

[20] Hevner K. Experimental studies of the elements of expression in music. Am. J. Psychol. 1936;48(2):22.

[21] Bradley M, Lang P. The international affective digitized sounds (2nd edition; iads-2): affective ratings of sounds and instruction manual. technical report b-3. Gainesville, Fl.: University of Florida, 2007.

[22] Friberg A, Breson R, Sundberg J. Overview of the KTH rule system for musical performance. Advances in Cognitive Psychology. 2006;2(2):145-161.

[23] Ramirez R, Vamvakousis Z. Detecting emotion from EEG signals using the emotive epoc device. Brain Informatics Lecture Notes in Computer Science: Springer; 2012:175-184.

[24] Schmidt LA, Trainor LJ. Frontal brain electrical activity (EEG) distinguishes valence and intensity of musical emotions. Cogn. Emot. 2001;15(4):13. doi:10.1080/02699930 04200187.

[25] Tenke CE, Kayser J. Reference-free quantification of EEG spectra: combining current source density (CSD) and frequency principal components analysis (fPCA). Clin. Neurophysiol. 2005;116(12):2826-2846. doi: 10.1016/j.clinph.2005.08.007. PubMed PMID: 16257577. 
[26] Vuoskoski JK, Thompson WF. Who enjoys listening to sad music and why? Music Perception: An Interdisciplinary Journal. 2012;29(3):311-317. doi:10.1525/ mp.2012.29.3.311.

[27] Salimpoor VN, Benovoy M, Longo G, et al. The rewarding aspects of music listening are related to degree of emotional arousal. PLoS ONE. 2009;4(10):e7487. doi: 10.1371/journal.pone.0007487. PubMed PMID: 19834599; PubMed Central PMCID: PMC2759002.

[28] Aftanas LI, Golocheikine SA. High-resolution EEG investigation of meditation.pdf. Neurosci. Lett. 2001;310(1):4.

[29] Giraldo, S and Ramirez R. Brain-activity-driven real-time music emotive control. in 3rd International Conference on Music \& Emotion (ICME3). 2013. Jyväskylä, Finland: Luck, G \& Brabant, O.

[30] Sammler D, Grigutsch M, Fritz T, et al. Music and emotion: electrophysiological correlates of the processing of pleasant and unpleasant music. Psychophysiology. 2007;44 (2):293-304. doi:10.1111/j.1469-8986.2007.00497.x.

[31] Aftanas LI, Reva NV, Savotina LN, et al. Neurophysiological correlates of induced discrete emotions in humans: an individually oriented analysis. Neurosci. Behav. Physiol. 2006;36(2):119-130. doi:10.1007/s11055-005-0170-6.

[32] Sebastiani L, Simoni A, Gemignani A, et al. Autonomic and EEG correlates of emotional imagery in subjects with different hypnotic susceptibility. Brain Res. Bull. 2003;60 (1-2):151-160. doi:10.1016/S0361-9230(03)00025-X.

[33] Dillman Carpentier FR. Potter RF. effects of music on physiological arousal: explorations into tempo and genre. Media Psychology. 2007;10(3):339-363.
[34] Daly I, Malik A, Hwang F, et al. Neural correlates of emotional responses to music: an EEG study. Neurosci. Lett. 2014;573:52-57. doi:10.1016/j.neulet.2014.05.003.

[35] Camurri A, Volpe G, Poli GD, et al. Communicating expressiveness and affect in multimodal interactive systems. IEEE Multimedia. 2005;12(1):43-53.

[36] Lipscomb SD. Cross-modal integration: synchronization of auditory and visual components in simple and complex media. The Journal of the Acoustical Society of America. $1999 ; 105(2)$.

[37] van der Zwaag MD, Westerink JHDM, van den Broek EL. Emotional and psychophysiological responses to tempo, mode, and percussiveness. Musicae Scientiae. 2011;15(2):250-269. doi:10.1177/1029864911403364.

[38] Hunt A, Kirk R. Mapping strategies for musical performance. In: Wanderley MM, Battier M, editors. Trends in gestural control of music. Ircam Centre Pompidou. 2000.

[39] Zander TO, Kothe C, Welke S, et al. Utilizing secondary input from passive brain-computer interfaces for enhancing human-machine interaction. Proceedings of the 5th International Conference on Foundations of Augmented Cognition Neuroergonomics and Operational Neuroscience: Held as Part of HCI International 2009; San Diego, CA. 1611171: Springer-Verlag; 2009. p. 759-771.

[40] Eaton J, Miranda E. Expanding on brain control: a realtime hybrid bcmi for drum machine combining active (SSVEP) and passive (affective response) brainwave control. 9th Conference on Interdisciplinary Musicology CIM14; 2014; Berlin, Germany. 